\title{
A Comparison of Two Methods for Choosing Repeatable Control Strategies for Kinematically Redundant Manipulators
}

\author{
Rodney G. Roberts Anthony A. Maciejewski \\ School of Electrical Engineering \\ Purdue University \\ West Lafayette, Indiana 47907
}

\begin{abstract}
A kinematically redundant manipulator is a robotic system that has more than the minimum number of degrees of freedom that are required for a specified task. Due to this additional freedom, control strategies may yield solutions which are not repeatable in the sense that the manipulator may not return to its initial joint conflguration for closed end effector paths. This paper presents two methods for choosing repeatable control strategies which minimize their distance from a non-repeatable inverse with desirable properties. The first method minimizes the integral norm of the difference of the desired inverse and a repeatable inverse. While this is the more appropriate criterion, it results in a difficult optimization. The second method, which minimizes the distance of the null vectors associated with the desired and the repeatable inverses, is somewhat easier to implement. As an illustrative example the pseudoinverse is approximated in a region of the joint space using both techniques.
\end{abstract}

\section{INTRODUCTION}

A robotic system can be described by its kinematic equation which relates the set of joint values of the manipulator to the position and orientation of the end effector in the workspace. If the location of the end effector is specified as an $m$-dimensional vector $\mathbf{x}$ then the kinematic equation can be written as

$$
\mathbf{x}=\mathbf{f}(\theta)
$$

where $\mathbf{f}$ is a smooth vector function and where $\theta$ is an $n$ dimensional vector of the joint angles. One of the popular techniques for controlling a manipulator is resolved motion rate control which calculates the joint velocities from the joint configuration and desired end effector velocity. The underlying equation is the Jacobian equation which, for the positional component, can be found by differentiating (1) to obtain

$$
\dot{\mathbf{x}}=J \dot{\theta}
$$

This work was supported by Sandia National Laboratories under contract number 18-4379B. Additional support was provided by the NEC Corporation and the TRW Foundation. where $\dot{\boldsymbol{x}}$ is the desired end effector velocity. The chief advantage of using the Jacobian for the motion control of a manipulator is that the Jacobian is a linear relationship between the joint velocities and the end effector velocities. At each point $\theta, J$ is an $m \times n$ matrix.

Kinematically redundant manipulators are robotic systems which possess more degrees of freedom than are required for a specified task so that $m<n$. This work will only consider the case of one degree of redundancy, i.e. when $n=m+1$. There are an infinite number of control strategies for redundant manipulators so that one can take advantage of this freedom by choosing a control strategy which will optimize some particular criterion. This work will consider generalized inverse strategies of the form

$$
\dot{\theta}=G \dot{\mathbf{x}}
$$

where $G$ satisfies $J G=I$ for nonsingular configurations. The elements of $G$ are functions of the joint configuration. This strategy may be chosen to locally minimize a given criterion function such as the least-squares minimum norm criterion on the joint velocities as in the case of the pseudoinverse solution

$$
\dot{\theta}=J^{+} \dot{\mathbf{x}}
$$

where $J^{+}$is the Moore-Penrose pseudoinverse of $J$. This control strategy locally minimizes the joint velocities of the manipulator subject to moving the end effector along a specified trajectory. Also popular in the robotics literature are weighted pseudoinverse solutions which locally minimize $\dot{\theta}^{T} Q \dot{\theta}$ for some positive definite weighting matrix $Q$.

Due to the additional freedom afforded to kinematically redundant manipulators, control strategies such as (3) may not be repeatable in the sense that closed trajectories in the work space are not necessarily mapped to closed trajectories in the joint space so that for cyclic tasks the manipulator will not necessarily return to its starting configuration. Klein and Huang [3] give a mathematical proof of this for the pseudoinverse control of a planar 3R manipulator. An elegant method of identifying control strategies which are repeatable is presented in a paper by Shamir and Yomdin [8]. This method determines repeatability by checking whether the Lie bracket 
of any two columns of the inverse is in the column space of $G$.

This work focuses on the generation of repeatable control strategies that are as close as possible to some desirable, but not repeatable, control. In Section II a set of repeatable control strategies, specified in terms of their associated augmenting vectors, is determined. Once such a set has been chosen, Sections III and IV describe methods for obtaining an optimal strategy. In Section III the inverse which is closest to a given desired inverse in an integral norm sense is chosen. This method, while conceptually simple, results in a difficult optimization problem. In order to simplify this optimization a second method is introduced in Section IV. Rather than working with the inverses themselves, this method selects an augmenting vector that minimizes its distance to the desired null vector on a subset of the joint space. Simulation results comparing these two methods are then presented in Section $\mathrm{V}$ using the standard planar $3 \mathrm{R}$ manipulator as an illustrative example. Finally, the conclusions of this work are presented in Section VI.

\section{GENERATING REPEATABLE CONTROL STRATEGIES}

In order to choose an optimal repeatable control strategy it is necessary to characterize those strategies which are repeatable in terms of the desired generalized inverse $G_{d}$ and a null space component. This will be done by considering the corresponding augmented Jacobian. At nonsingular configurations any generalized inverse $G$ can be calculated by inverting an augmented Jacobian of the form

$$
J_{v}=\left[\begin{array}{c}
J \\
\cdots \\
v^{T}
\end{array}\right]
$$

where $\mathbf{v}$ is a null vector of $G^{T}$. The corresponding control strategy is found by taking the first $m$ columns of the inverse of $J_{v}^{-1}$ which is given by

$$
J_{\mathbf{v}}^{-1}=\left[\begin{array}{lll}
G_{d}+\hat{\mathbf{n}}_{J} \mathbf{w}^{T} & \vdots & \frac{\hat{\mathbf{n}}_{J}}{\hat{\mathbf{n}}_{J} \cdot \mathbf{v}}
\end{array}\right]
$$

where $\hat{\mathbf{n}}_{J}$ is a unit length null vector and

$$
\mathbf{w}=\frac{-G_{d}^{T} \mathbf{v}}{\hat{\mathbf{n}}_{J} \cdot \mathbf{v}}
$$

It is a well known fact that one can obtain a repeatable control strategy for a manipulator with one degree of redundancy by inverting a Jacobian which has been augmented with a gradient [7]. Thus the augmented task space approach is one commonly used technique for resolving manipulator redundancy [6]. For the extended Jacobian [1], the augmenting vector is given by the gradient of $\nabla g \cdot n_{J}$ where $g$ is some criterion function of $\theta$. By including this additional function the manipulator acts "mathematically" like a nonredundant manipulator assuming that the rows of $J$ and $\mathbf{v}$ are linearly independent. A set of these gradients can be used to define a class of control strategies which are repeatable in simplyconnected, singularity-free domains [2].

An example of a set of augmenting vectors which yield repeatable control strategies is the span of $N$ linearly independent gradient functions $\left\{\mathbf{v}_{1}, \mathbf{v}_{2}, \ldots, \mathbf{v}_{N}\right\}$. For this case the augmenting vectors would take on the form $\mathbf{v}=\sum_{i=1}^{N} a_{i} v_{i}$ where each $a_{i}$ is a real constant. Several considerations should be made in choosing such a basis. One should be careful to select the gradient functions to be linearly independent from the row space of the Jacobian since failure to do so will result in a singular augmented Jacobian. Secondly it should be noted that all nonzero multiples of an augmenting vector result in the same control. Thus choosing an optimal augmenting vector becomes a constrained optimization problem in which each augmenting vector is normalized. Such a normalization can be done for example by requiring that $\sum_{i=1}^{N} a_{i}^{2}=1$.

\section{THE NEAREST REPEATABLE CONTROL STRATEGY}

Now that a class of repeatable strategies has been defined, it is possible to choose an optimal solution. The nearest repeatable control strategy is defined as the control strategy from a set of repeatable strategies which is nearest to some desired non-repeatable strategy in some region of the joint space. A particular example of a set of repeatable strategies was given above as a parametrization of the span of a set of gradient functions. Once a set of repeatable control strategies has been chosen, a measure must be decided upon so that an optimal strategy can be determined. The criterion used in this work will be a measure of the distance between the desired inverse $G_{d}$ and the repeatable inverse $G_{r}$ defined by the following norm

$$
\left\|G_{r}-G_{d}\right\|_{\Omega}^{2}=\int_{\Omega}\left\|G_{r}-G_{d}\right\|_{2}^{2} d \theta
$$

where $\Omega$ is a simply-connected, singularity-free subset $\Omega$ of the joint space. Equation (8) provides a measure of the closeness of two inverses on some important subset $\Omega$ of the joint space. The subset $\Omega$ may be chosen based on some optimal configuration at which one would like the manipulator to operate. From (6) it follows that the euclidean matrix norm of the difference between the inverses $G_{r}$ and $G_{d}$ is

$$
\left\|G_{r}-G_{d}\right\|_{2}=\left\|\hat{\mathbf{n}}_{J} \mathbf{w}\right\|_{2}=\|\mathbf{w}\|_{2} .
$$

Thus the measure given in (8) for a repeatable inverse and a desired inverse becomes

$$
\left\|G_{r}-G_{d}\right\|_{\Omega}^{2}=\int_{\Omega}\|\mathbf{w}\|_{2}^{2} d \theta
$$


where $\mathbf{w}$ is given by (7).

Only for very simple cases can one analytically optimize (10) to find a repeatable inverse which is near a desired inverse [4]. For more complicated manipulators the optimization must be performed numerically using a basis of allowable augmenting vectors. Suppose that a set of $N$ linearly independent gradients $\left\{\mathbf{v}_{1}, \mathbf{v}_{2}, \ldots, \mathbf{v}_{N}\right\}$ has been chosen as in Section II. The span of this set consists of all vectors of the form

$$
\mathbf{v}=\sum_{i=1}^{N} a_{i} \mathbf{v}_{i}
$$

where the $a_{i}$ are real scalar constants. It is easy to verify that (11) is a gradient due to the linearity of the differential operator. Combining equations (7), (10), and (11) yields

$$
\left\|G_{r}-G_{d}\right\|_{\Omega}^{2}=\int_{\Omega} \frac{\sum_{i=1}^{N} \sum_{j=1}^{N} a_{i} a_{j} \mathbf{v}_{i}^{T} G_{d} G_{d}^{T} \mathbf{v}_{j}}{\left(\sum_{k=1}^{N} a_{k} \hat{\mathbf{n}}_{J} \cdot \mathbf{v}\right)^{2}} d \theta
$$

which is a criterion function of the coefficients $a_{i}$. As discussed in Section II the coefficients should be normalized in some fashion since any scaled version of the augmenting vector will yield the same inverse. The problem then becomes an $(N-1)$-dimensional optimization. One final consideration is the possible existence of algorithmic singularities for certain combinations of $a_{i}$ in the domain $\Omega$. Such combinations of coefficients should be avoided when determining an optimal solution.

\section{NULL SPACE APPROXIMATION}

The above optimization can be rather difficult since the criterion function is in general highly nonlinear. Even when a minimum is obtained it is difficult to determine whether it is in fact a global minimum. A more computationally efficient optimization can be developed by considering the problem in terms of the function space $\mathcal{L}_{2}(\Omega)$. The space $\mathcal{L}_{2}(\Omega)$ is a separable Hilbert space consisting of the set of Lebesgue measurable functions $\mathbf{u}: \Omega \rightarrow \mathbb{R}^{n}$ which satisfy $\int_{\Omega}\|\mathbf{u}\|_{2}^{2} d \theta<\infty$ along with an inner product defined by

$$
<\mathbf{u}, \mathbf{v}>_{\Omega}=\int_{\Omega} \mathbf{u} \cdot \mathbf{v} d \theta
$$

for any two vector functions $\mathbf{u}, \mathbf{v}$ in $\mathcal{L}_{2}(\Omega)$ where $\mathbf{u} \cdot \mathbf{v}$ is the standard dot product and $\int_{\Omega} d \theta$ is the Lebesgue integral on $\Omega \subset \mathbb{R}^{n}$. The corresponding integral norm

$$
\|\mathbf{u}\|_{\Omega}=\left[\int_{\Omega}\|\mathbf{u}\|_{2}^{2} d \theta\right]^{\frac{1}{2}}
$$

will be used as a measure of the distance between vector functions on $\Omega$. Since $\mathcal{L}_{2}(\Omega)$ is a Hilbert space it follows that for any closed subspace $U$ and any $w \in \mathcal{L}_{2}(\Omega)$ there exists a unique $u \in U$ such that $\|\mathbf{u}-\mathbf{w}\|_{\Omega}$ is minimal. The measure of the distance between two subsets $V$ and $W$ of $\mathcal{L}_{2}(\Omega)$ is defined to be $\operatorname{dist}(U, W)=\inf \left\{\|\mathbf{v}-\mathbf{w}\|_{\Omega} \mid \mathbf{v} \in\right.$ $V, \mathbf{w} \in W\}$.

The criterion used in this section is to minimize the distance between the null vectors of the desired and the repeatable inverses. In particular this work is concerned with minimizing the distance between a subset $\mathcal{N}_{0}$ of the null space $\mathcal{N}$ of $G_{d}$ and a space of allowable augmenting vectors $\mathcal{V}$. This subset $\mathcal{N}_{0}$ is the set of null vector functions which are normalized in the norm $\|\cdot\| \Omega$. A desirable gradient $\mathbf{v}^{*}$ will have the property that it minimizes $\inf _{\mathbf{n} \in \mathcal{N}_{0}}\left\|\mathbf{v}^{*}-\mathbf{n}\right\|_{\Omega}$. For the space $\mathcal{N}_{0}$ each element can be written in the form $\alpha \hat{\mathbf{n}}_{G}$ for nonsingular configurations where $\alpha$ is any continuous function, $\hat{\mathbf{n}}_{G}$ is the unit null vector associated with $G_{d}$, and $\left\|\alpha \hat{\mathbf{n}}_{G}\right\|_{\Omega}=1$. Thus if $\Omega$ does not contain any singularities $\mathcal{N}_{0}(\Omega)=\left\{\alpha \hat{\mathbf{n}}_{G} \mid \int_{\Omega} \alpha^{2} d \theta=1\right\}$.

As above it will be assumed that the set of allowable augmenting vectors $\mathcal{V}_{N}$ is given by a basis of gradient functions $\left\{\mathbf{v}_{1}, \mathbf{v}_{2}, \cdots, \mathbf{v}_{N}\right\}$ with the further requirement that this basis be orthonormal in $\mathcal{L}_{2}(\Omega)$. With this restriction the Projection Theorem guarantees that the element of $\mathcal{V}_{N}$ which is closest to some arbitrary vector function $\mathbf{n}$ in $\mathcal{L}_{2}(\Omega)$ is given by

$$
\mathbf{v}^{*}(\mathbf{n})=\sum_{i=1}^{N}<\mathbf{n}, \mathbf{v}_{i}>_{\Omega} \mathbf{v}_{i}
$$

which is simply the orthogonal projection of $\mathbf{n}$ onto $\mathcal{V}_{N}$. Thus for any vector function $\mathbf{n} \in \mathcal{N}_{0}$ the element in $\mathcal{V}_{N}$ which minimizes $\|\mathbf{v}-\mathbf{n}\|_{\Omega}$ is the orthogonal projection of n onto $\mathcal{V}_{N}$.

Now that an appropriate subspace has been defined, one can choose an augmenting vector from this set which minimizes its distance from $\mathcal{N}_{0}$, the space of null vectors which are of unit length in the norm $\|\cdot\|_{\Omega}$. This is done by finding the appropriate $\alpha^{*}$ and the $\mathbf{v}$ in $\mathcal{V}_{N}$ which minimizes $\left\|\alpha^{*} \hat{\mathbf{n}}_{G}-\mathbf{v}\right\|_{\Omega}^{2}$ with $\int_{\Omega} \alpha^{2} d \theta=1$. This minimization will be done in two steps. First the form of the $\alpha$ 's which is closest to $\mathcal{V}_{N}$ will be stated. Then the corresponding $\mathbf{v}$ in $\mathcal{V}_{N}$ for each of the candidate $\alpha$ 's is calculated. Finally the minimal pair is chosen from these candidates.

The $\mathbf{n}$ in $\mathcal{N}_{0}$ which is closest to a $\mathbf{v}$ in $\mathcal{V}_{N}$ is characterized in terms of its corresponding $\alpha$ by the following proposition:

Proposition 1 Let $\mathbf{v}=\sum_{i=1}^{N} c_{i} \mathbf{v}_{i}$ be a fixed vector function in $\mathcal{V}_{N}$ and suppose that $\sum_{i=1}^{N} c_{i} \mathbf{v} \cdot \hat{\mathbf{n}}_{G} \neq 0$. Suppose $\alpha^{*}=\arg \min _{\alpha \in \mathcal{A}}\left\|\alpha \hat{\mathbf{n}}_{G}-\sum_{i=1}^{N} c_{i} \mathbf{v}_{i}\right\|_{\Omega}^{2}$. Then there exists a constant $K$ such that

$$
\alpha^{*}=K \sum_{i=1}^{N} c_{i} \mathbf{v}_{i} \cdot \hat{\mathbf{n}}_{G} .
$$




\section{Proof See [5]}

Thus the candidate $\alpha$ 's are of the form given in (16).

For each $\mathbf{n} \in \mathcal{N}_{0}$ the corresponding $\mathbf{v} \in \mathcal{V}_{N}$ which is closest to $\mathbf{n}$ is the orthogonal projection of $\mathbf{n}$ onto $\mathcal{V}_{N}$. Let $\mathbf{v}(\alpha)$ denote the orthogonal projection of $\alpha \hat{\mathbf{n}}_{G}$ onto $\mathcal{V}_{N}$. Thus the problem now becomes that of minimizing

$$
\left\|\alpha \hat{\mathbf{n}}_{G}-\mathbf{v}(\alpha)\right\|_{\Omega}
$$

By Proposition 1 a minimal $\alpha$ would have the form given in (16). Thus there exist $b_{1}, \ldots, b_{N}$ such that

$$
\alpha=\sum_{j=1}^{N} b_{j} \mathbf{v}_{j} \cdot \hat{\mathbf{n}}_{G}
$$

Let $a_{i}(\alpha)$ be the generalized Fourier coefficient of $\alpha \hat{\mathbf{n}}_{G}$ corresponding to $\mathbf{v}_{i}$ which is given by $\left\langle\alpha \hat{\mathbf{n}}_{G}, \mathbf{v}_{i}>_{\Omega}\right.$. From (18) it follows that

$$
a_{i}(\alpha)=\sum_{j=1}^{N} b_{j} \int_{\Omega}\left(\mathbf{v}_{i} \cdot \hat{\mathbf{n}}_{G}\right)\left(\mathbf{v}_{j} \cdot \hat{\mathbf{n}}_{G}\right) d \theta
$$

In order to make the presentation clearer some vector notation is introduced. Let $\mathbf{a}=\left[a_{1}(\alpha), \ldots, a_{N}(\alpha)\right]^{T}$ correspond to the series $\sum_{j=1}^{N} a_{j} \mathbf{v}_{j}$, let $\mathbf{b}=\left[b_{1}, \ldots, b_{N}\right]$, and let the matrix $M$ be the Gramian matrix defined by

$$
M_{i j}=\int_{\mathbf{\Omega}}\left(\mathbf{v}_{\boldsymbol{i}} \cdot \hat{\mathbf{n}}_{G}\right)\left(\mathbf{v}_{j} \cdot \hat{\mathbf{n}}_{G}\right) d \theta
$$

Using this notation (19) becomes

$$
\mathbf{a}=M \mathbf{b} .
$$

In order for $\left\|\alpha \hat{\mathbf{n}}_{G}\right\|_{\Omega}=1$ there is a restriction on $\mathbf{b}$. Integrating the square of (18) yields

$$
\int_{\mathbf{n}} \alpha^{2} d \theta=\sum_{i=1}^{N} \sum_{j=1}^{N} \int_{\mathbf{\Omega}}\left(\mathbf{v}_{i} \cdot \hat{\mathbf{n}}_{G}\right)\left(\mathbf{v}_{j} \cdot \hat{\mathbf{n}}_{G}\right) d \theta b_{i} b_{j}
$$

which is equal to one, which in vector notation becomes

$$
\mathbf{b}^{T} M \mathbf{b}=1 .
$$

Now $\left\|\alpha \hat{\mathbf{n}}_{G}-\mathbf{v}(\alpha)\right\|_{\Omega}^{2}=1-\mathbf{a}^{T} \mathbf{a}$. Thus it is important to maximize $\mathbf{a}^{T}$ a subject to (23). This maximum occurs when $\mathbf{a}$ and $\mathbf{b}$ are eigenvectors of $M$ associated with its largest eigenvalue. Since $M$ is a symmetric positive semidefinite matrix, a can be found from the singular value decomposition of $M$. The vector a would simply be $\sqrt{\sigma_{1}} \mathbf{u}_{1}$ where $\sigma_{1}$ is the largest singular value and $\mathbf{u}_{1}$ is its corresponding singular vector. It is this vector of generalized Fourier coefficients which minimizes the distance to $\mathcal{N}_{0}$.

\section{AN ILLUSTRATIVE EXAMPLE}

In order to illustrate the two methods discussed above, consider the three-link manipulator shown in Fig. 1 which has links of unit length. The Jacobian for this particular manipulator is

$J=\left[\begin{array}{ccc}-s \theta_{1}-s \theta_{12}-s \theta_{123} & -s \theta_{12}-s \theta_{123} & -s \theta_{123} \\ c \theta_{1}+c \theta_{12}+c \theta_{123} & c \theta_{12}+c \theta_{123} & c \theta_{123}\end{array}\right]$

where $\theta_{i j}=\theta_{i}+\theta_{j}$. Note that the manipulator described by (24) is given in terms of the more realistic relative angles as opposed to the simpler absolute angles which are frequently used in work concerning repeatability. For a simply-connected, singularity-free subset of the joint space the unit null vector $\hat{\mathbf{n}}_{J}$ for this manipulator can be continuously and uniquely defined up to a multiple of -1 . In particular it can be obtained by normalizing the cross product of the two rows of the Jacobian:

$$
\hat{\mathbf{n}}_{J}=\frac{1}{\Delta}\left[\begin{array}{c}
s \theta_{3} \\
-s \theta_{3}-s \theta_{23} \\
s \theta_{2}+s \theta_{23}
\end{array}\right]
$$

where $\Delta=\sqrt{s^{2} \theta_{3}+\left(s \theta_{3}+s \theta_{23}\right)^{2}+\left(s \theta_{2}+s \theta_{23}\right)^{2}}$. For this example the desired optimization criterion will be to minimize the norm of the joint angle velocities. The exact solution for this criterion is given by the pseudoinverse of the Jacobian; however, it is well-known that the pseudoinverse is not repeatable [3]. The task here is to approximate the pseudoinverse using the two techniques discussed previously. Since the pseudoinverse cannot be accurately approximated over the entire joint space, one must select a simply-connected, singularity-free region $\Omega=I_{1} \times I_{2} \times I_{3}$ where $I_{i}=\left[a_{i}, b_{i}\right]$ with $a_{i}<b_{i}$ for $i=1,2,3$. The boundaries of this region can be chosen based on the particular physical constraints of the manipulator or the requirements of the task being performed. For this particular example let the set $\Omega$ be given by the cube $[\pi / 4,3 \pi / 4]^{3}$.

The first step is to choose a set of augmenting vectors which are gradients so that the corresponding inverses will be repeatable. In addition to being a set of gradients the second method requires that this set be given in terms of an orthonormal basis contained in $\mathcal{L}_{2}(\Omega)$. In order to compare the two methods, the same basis will be used. A simple example of such a basis is given by

$$
\left\{\kappa e_{1}, \kappa e_{2}, \kappa e_{3}\right\}
$$

where $\kappa=8 / \pi^{3}$ and where $e_{i}$ represents the standard basis for $\mathbb{R}^{3}$. An advantage of the nearest repeatable method is that it only requires a linearly independent basis as opposed to an orthogonal one as in the case of the 
null space approximation method. However, this advantage is overshadowed by the difficulty of the optimization criterion associated with the first method.

The optimization for the nearest repeatable trajectory was done numerically by minimizing (12) as outlined in Section III. The optimal augmenting vector was found to be

$$
\mathbf{v}=\left[\begin{array}{lll}
-0.8381 & 0.2065 & -0.5048
\end{array}\right]^{T} .
$$

For the null space approximation method the Gramian matrix $M$ was calculated from (20) resulting in

$$
M=\left[\begin{array}{ccc}
0.4275 & -0.2559 & 0.2579 \\
-0.2559 & 0.2844 & -0.2814 \\
0.2579 & -0.2814 & 0.2881
\end{array}\right]
$$

The optimal augmenting vector for this optimization is found by calculating the singular value decomposition of $M$, which is given by $U \Sigma U^{T}$ since $M$ is symmetric, where

$$
U=\left[\begin{array}{ccc}
-0.6367 & -0.7711 & -0.0013 \\
0.5434 & -0.4498 & 0.7088 \\
-0.5472 & 0.4506 & 0.7054
\end{array}\right]
$$

ard where the diagonal elements of the matrix $\Sigma$ are given by $(0.868,0.128,0.005)$. The singular vector associated with the largest singular value represents the desired augmenting vector so that for this method

$$
\mathbf{v}=\left[\begin{array}{lll}
-0.6367 & 0.5434 & -0.5472
\end{array}\right]^{T} .
$$

Clearly by comparing (27) with (30) one can see that these two techniques have resulted in two different repeatable inverses. A comparison of the performance of these inverses relative to the desired non-repeatable pseudoinverse was performed for the square end effector trajectory shown in Fig. 1. The norm of the joint angle velocities for both the repeatable inverses as well as the pseudoinverse are shown in Fig. 2. Note that for the initial phase of the trajectory from $A$ to $B$ the performance of the nearest repeatable inverse is nearly identical to that of the pseudoinverse with the null space approximation technique being comparable. However, when the direction of the desired end effector velocity is changed abruptly at $B$, the performance changes radically. This clearly illustrates that the resulting performance is not only linked to the configuration of the manipulator which determines the inverse but also to the characteristics of the specified end effector velocity. During the trajectory from $B$ to $C$ the nearest repeatable inverse is clearly a better approximation to the pseudoinverse than the inverse obtained from the null space approximation technique. After this part of the trajectory there is little point in comparing the repeatable inverses to the pseudoinverse since there is no longer a correlation between the respective manipulator configurations. This is graphically illustrated in Fig. 3 which

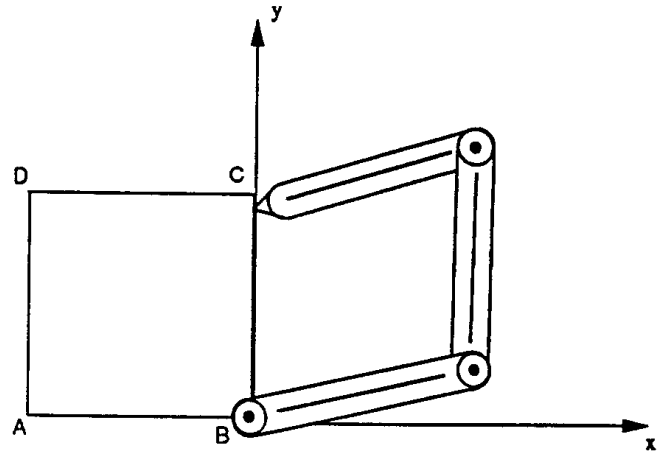

Fig. 1 The planar 3R manipulator and the square end effector trajectory used in the illustrative example. The manipulator starts at $A$ with an initial configuration of $[\pi / 2 \pi / 2 \pi / 2]$.

gives a measure of the difference between the configurations obtained using the repeatable inverses and that obtained using the pseudoinverse. Note that as was inferred from the norm curves, the configurations of the manipulator for the three techniques are comparable for the initial phase of the trajectory. After point B, however, the configuration of the manipulator resulting from pseudoinverse control diverges from the repeatable controls and results in non-repeatable behavior. In contrast, the two repeatable inverses are constrained to match configurations at A so that there must be a closer correspondence in the resulting joint space trajectories.

\section{CONCLUSIONS}

The contribution of this work is a comparison of two techniques for generating repeatable generalized inverses which are close to some arbitrary given generalized inverse. Both techniques characterize a set of repeatable inverses by the gradient functions which correspond to their null vectors. The difference in the two methods is in the optimization on which each relies. The first technique minimizes the integral norm of the difference between the repeatable inverse and the desired inverse over a subset $\Omega$. While this gives a more appropriate measure of the two techniques it is certainly the more difficult one to calculate. The second technique tries to most nearly match the null vector of the desired inverse and relies on using a set of orthonormal basis functions to describe a set of possible gradient functions. The optimal coefficients for these basis functions can be easily determined by calculating the singular vector associated with the maximum singular value of the Gramian matrix. It was shown that both techniques can only provide accurate approximations to the desired inverse on limited portions of the workspace. 


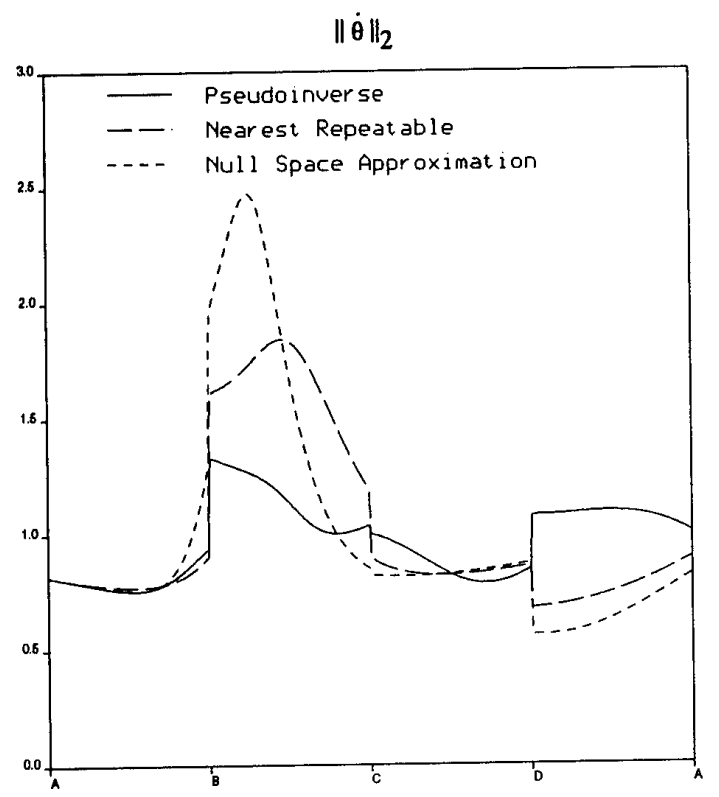

Fig. 2 A comparison of the norm of the joint velocities for the optimal desired non-repeatable inverse (pseudoinverse) versus the two different repeatable inverses for the trajectory shown in Fig. 1.

\section{REFERENCES}

[1] J. Baillieul, "Kinematic programming alternatives for redundant manipulators," in Proc. IEEE Int. Conf. Robotics Automat. (St. Louis, MO, March 25-28, 1985), pp. 722-728.

[2] D.R. Baker and C.W. Wampler II, "On the inverse kinematics of redundant manipulators", Int. $J$. Robotics Res., vol. 7, no. 2, pp. 3-21, March/April 1988.

[3] C.A. Klein and C.H. Huang, "Review of pseudoinverse control for use with kinematically redundant manipulators," IEEE Trans. Syst., Man Cyber., vol. SMC-13, no. 3, pp. 245-250, March/April 1983.

[4] R.G. Roberts and A.A. Maciejewski, "Nearest optimal repeatable control strategies for kinematically redundant manipulators," IEEE Trans. Robotics Automat., vol. 8, 1992.

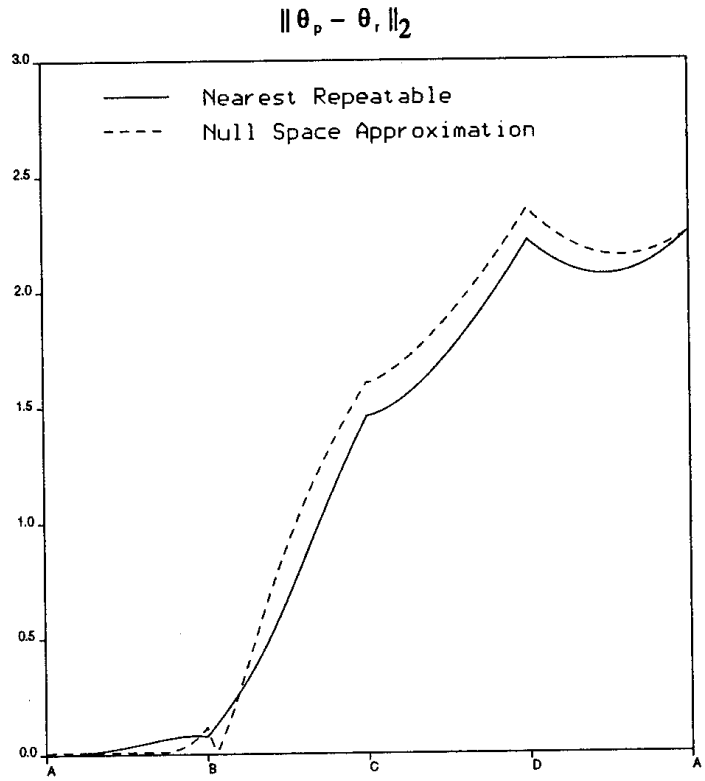

Fig. 3 A comparison of the deviations in joint configuration while using the two repeatable controls as compared with the pseudoinverse control.

[5] R.G. Roberts and A.A. Maciejewski, "Repeatable generalized inverse control strategies for kinematically redundant manipulators," in Proc. 30th Conf. Decision Contr. (Brighton, UK, Dec. 11-13, 1991), pp. 2428-2434.

[6] H. Seraji, "Configuration control of redundant manipulators: Theory and implementation," IEEE Trans. Robotics Automat., vol. 5, no. 4, pp. 472-490, Aug. 1989.

[7] T. Shamir, "Remarks on some dynamical problems of controlling redundant manipulators," IEEE Trans. Automat. Contr., vol. 35, no.3, pp. 341-344, March 1990.

[8] T. Shamir and Y. Yomdin, "Repeatability of redundant manipulators: mathematical solution of the problem," IEEE Trans. Automat. Contr., vol. 33, no. 11, pp. 1004-1009, Nov. 1988. 